\title{
A blind comparison of the effectiveness of endoscopic ultrasonography and endoscopy in staging early gastric cancer
}

\author{
H Yanai, T Noguchi, S Mizumachi, H Tokiyama, H Nakamura, M Tada, K Okita
}

\begin{abstract}
BackgroundlAims-Endoscopic ultrasonography is expected to be useful for invasion depth staging of early gastric cancer. A prospective blind study of the staging characteristics of endoscopy and endoscopic ultrasonography for early gastric cancer was performed.

Methods-Findings of endoscopy and endoscopic ultrasonography using a $20 \mathrm{MHz}$ thin ultrasound probe were independently reviewed and the results of 52 early gastric cancer lesions analysed.

Results-The overall accuracy rates in invasion depth staging of early gastric cancer were $63 \%$ for endoscopy and $71 \%$ for endoscopic ultrasonography. No statistically significant differences were observed in overall accuracy. Endoscopic ultrasonography tended to overstage, and lesions that were classified as mucosal cancer by endoscopic ultrasonography were very likely $(95 \%)$ to be limited to the mucosa on histological examination. All 16 lesions staged as mucosal cancer independently but coincidentally by both methods were histologically limited to the mucosa.

Conclusions-Endoscopic ultrasonography is expected to compensate for the understaging of lesions with submucosal invasion that are endoscopically staged as mucosal cancer.

(Gut 1999;44:361-365)
\end{abstract}

Keywords: early gastric cancer; endoscopic ultrasonography; endoscopy

The first use of the ultrasonic endoscope was reported in 1980, and the use of endoscopic ultrasonography (EUS) has become widespread for examination of the biliary tract, gall bladder, pancreas, and gastrointestinal tract. ${ }^{1-5}$ In the field of stomach diseases, the importance of tumour staging of gastric cancer has been increasing with the development of the endoscopic resection technique. ${ }^{6-8}$ EUS has been expected to be useful for invasion depth staging of early gastric cancer. ${ }^{9-13}$ Although EUS using the appropriate instrument $(7.5-12.5 \mathrm{MHz})$ is useful for advanced gastric cancer, optical control of such a large instrument for small lesions is not easy and the resolution is insufficient for the precise observation of superficial lesions. The recent introduction of high frequency thin endoscopic ultrasound probes has enabled us to perform target scanning with high resolution of even very small gastric cancer lesions under endoscopic control. ${ }^{14-16}$

Conventional endoscopy has been the most useful diagnostic modality for early gastric cancer. ${ }^{17}{ }^{18}$ Unfortunately, there are only a few reports on the relation between the tumour invasion depth staging characteristics of endoscopy and EUS. Therefore the true role of EUS in early gastric cancer depth staging is still unclear. To obtain fundamental data to answer such questions, we previously performed a retrospective non-blinded pilot study of the staging characteristics of endoscopy and EUS in 108 early gastric cancer lesions. ${ }^{19}$ The overall accuracy rates for staging depth of invasion for endoscopy and EUS were 72.2 and $64.8 \%$ respectively. Staging characteristics with regard to understaging and overstaging of the two methods were significantly different. EUS showed a higher overstaging rate. Lesions that were classified by both endoscopy and EUS as being limited to the mucosa were very likely $(92.2 \%)$ to be found to be limited to the mucosa on histological examination. To confirm the pilot study results, we carried out a prospective blind study.

\section{Materials and methods}

PATIENTS

Patients referred to the First Department of Internal Medicine, Yamaguchi University School of Medicine or Hofu Institute of Gastroenterology with early gastric cancer lesions found by previous endoscopy and biopsy were included in the study of simultaneous invasion depth staging using endoscopy and thin probe EUS. Lesions diagnosed as obviously advanced cancer (cancer invading the muscularis propria or deeper) by previous endoscopy were excluded. Before obtaining histological results, we sent endoscopy and EUS photoprints (four to eight prints) separately to an experienced endoscopist (S M) and endosonographer ( $\mathrm{T} \mathrm{N}$ ) blinded to any other information. Between August 1996 and September 1997, 59 consecutive patients previously diagnosed as having early gastric cancer underwent invasion depth staging using endoscopy and a high frequency thin EUS probe. Seven of the 59 patients were excluded: one refused surgical operation; in two the histological examination of endoscopically resected specimens finally confirmed their lesions as adenoma; in four the specimens were not

Abbreviation used in this paper: EUS, endoscopic ultrasonography.
Medicine, 1144 Kogushi,

Ube, Yamaguchi 755-8505, Japan.

Accepted for publication 2 September 1998 
Table 1 Macroscopic classifications of endoscopically diagnosed early gastric cancer lesions

\begin{tabular}{ll}
\hline Macroscopic type & Lesions $(n=52)$ \\
\hline Type 0I (protruded) & 2 \\
Type OII (superficial) & 8 \\
IIa (superficial elevated) & $2^{\star}$ \\
IIa+IIc & $29^{\star}$ \\
IIc (superficial depressed) & 10 \\
IIc+III & 1 \\
Type 0III (excavated) & \\
\hline
\end{tabular}

${ }^{\star}$ Included one lesion found to be advanced gastric cancer.

adequate for histological confirmation of invasion depth. Consequently, 52 lesions were analysed. There were 40 men and 12 women (mean age 66.9 years, range 32 to 81 ). The superficial depressed type of lesion was dominant (table 1). Endoscopic treatment (strip biopsy $^{6}$ ) was performed in 19 cases, and 33 patients had surgery. There were two lesions for which the final histological diagnosis was advanced gastric cancer.

ENDOSCOPY

The endoscope used in this study was a GIF-2T200 forward viewing type electronic panendoscope (Olympus, Tokyo, Japan). The GIF-2T200 has dual forceps channels and allows frequent water infusion and suction during thin probe EUS scanning. Endoscopic staging for tumour invasion depth in early gastric cancer was performed following a standard protocol. ${ }^{17}{ }^{19}$ Lesions that protruded from the mucosa with a smooth surface were classified as mucosal (endoscopy-mucosal). Lesions with a shallow and smooth surfaced depression were also classified as endoscopy-mucosal. Lesions considered to exhibit submucosal invasion (endoscopy-submucosal) were those that showed a more uneven base, with an irregularly shaped nodule, or those with folds that were enlarged.

ENDOSCOPIC ULTRASONOGRAPHY

The $20 \mathrm{MHz}$ thin endoscopic ultrasound probes used in this study were equipped with switchable radial and linear scanning modes SP-501 (23 lesions) and SP-701 (24 lesions) interfaced to a Sonoprobe System (Fujinon, Omiya, Japan) and radial scanning mode UM-3R (five lesions; Olympus). The probes yielded high quality cross sectional images of the mucosa and submucosa and were easily directed to the small cancer lesions under the direct vision of the endoscopist. ${ }^{14-16}$ The EUS images were interpreted with regard to tumour invasion according to the five layer architecture of the gastric wall (figs 1-3), and lesions were classified as mucosal (EUS-mucosal), submucosal (EUS-submucosal), or advanced (tumour had invaded the muscularis propria or deeper, EUS-advanced). On EUS, the gastric mucosa is visualised as a combination of the first hyperechoic and second hypoechoic layers, and the submucosa corresponds to the third hyperechoic layer. The muscularis propria is visualised as the fourth hypoechoic layer, and the fifth hyperechoic layer is the serosa including the subserosa. ${ }^{2021}$ According to our previous reports, the fine hypoechoic
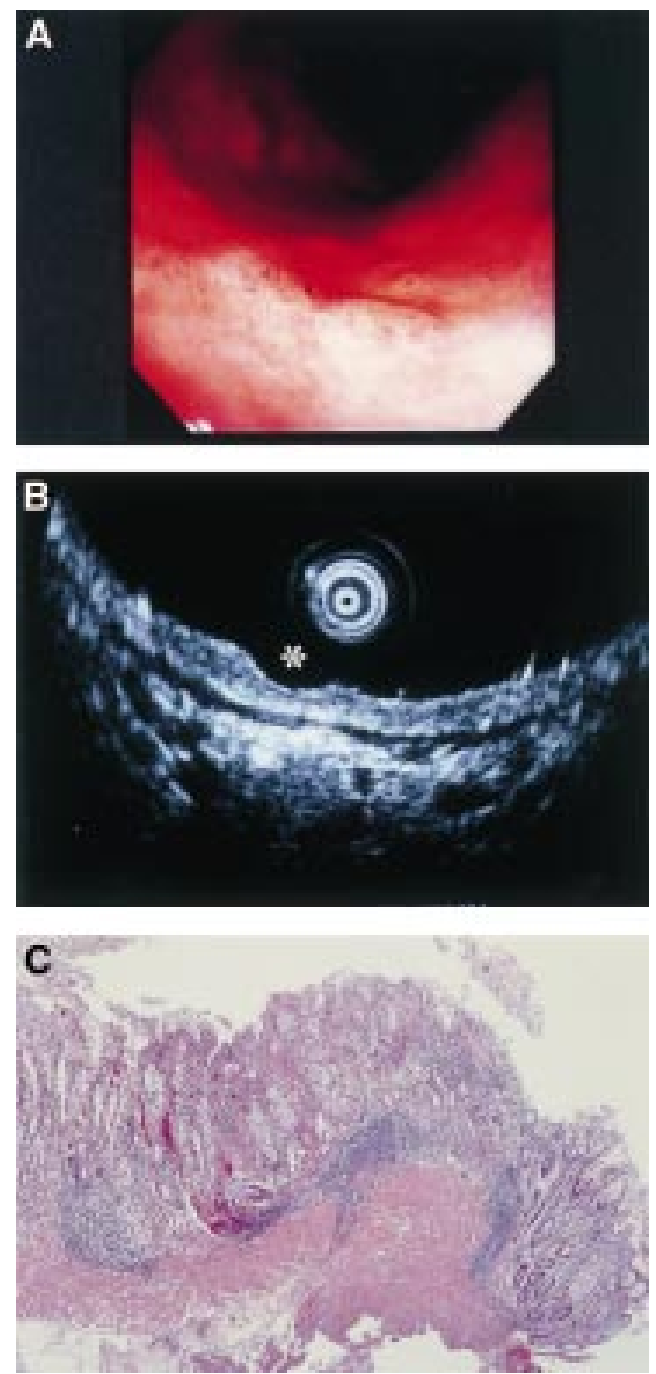

Figure 1 A superficial depressed type early gastric cancer located in the lower gastric body. Endoscopy $(A)$ and endoscopic ultrasonography (B, asterisk) reviewers independently but coincidentally staged this as mucosal. Histological examination $(C)$ of its endoscopically resected strip biopsy specimen also proved that it was mucosal cancer. In this case, both methods staged correctly.

layer between the second and third layers is considered to correspond to the muscularis mucosae. ${ }^{15}$ When the muscularis mucosae and intermuscular interface of the muscularis propria are visualised, the normal gastric wall is observed as a nine layered structure. ${ }^{19}$ The muscularis mucosae is expected to be visualised in almost $30 \%$ of cases in the stomach and the oesophagus. ${ }^{162223}$

\section{HISTOLOGY}

Surgically or endoscopically resected specimens were systematically examined by the regular procedure specified in the Japanese Classification of Gastric Carcinoma. ${ }^{24}$ Specimens were fixed and stained with haematoxylin and eosin, and the depth of invasion was classified as mucosal, submucosal, or advanced (the tumour had invaded the muscularis propria or deeper).

STATISTICAL ANALYSIS

Data were analysed using the $\chi^{2}$ test. A $p$ value of less than 0.05 was considered significant. 

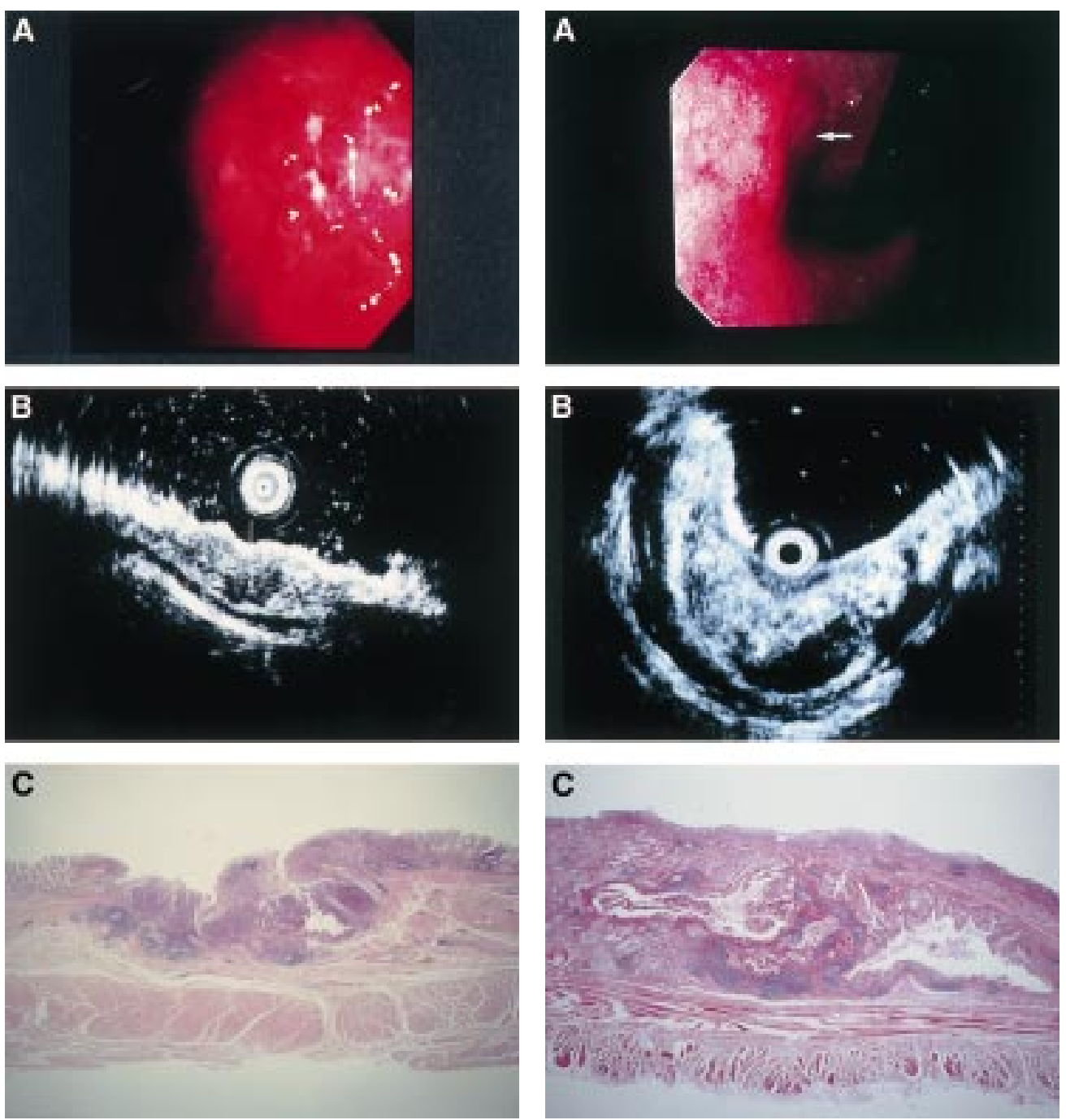

Figure 2 A superficial depressed type early gastric cancer located in the middle gastric body. This case was

understaged by endoscopy. The endoscopy reviewer observed that the surface of the depression was not so rough, and staged it as endoscopy-mucosal (A). In contrast, the endoscopic ultrasonography (EUS) reviewer observed hypoechoic tumorous invasion and irregular narrowing of the third layer ( $B$, white arrow). He staged it as depth EUS-submucosal. Histological findings of a surgically resected specimen corresponded well to the EUS image analysis (C).

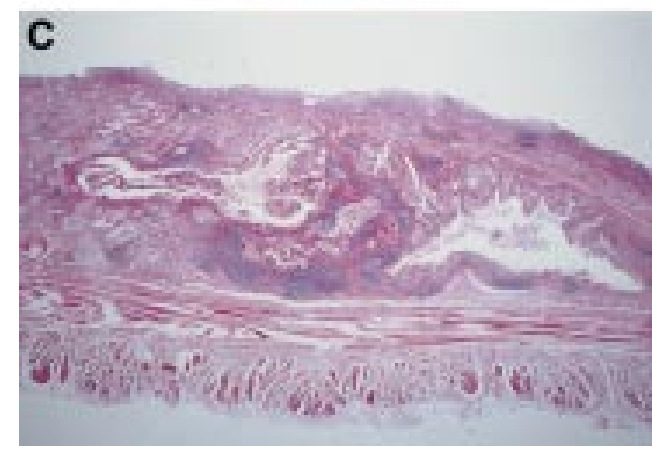

Figure 3 A superficial depressed type early gastric cancer located in the cardia. This was also understaged by

endoscopy. As the lesser curvature of the cardia is a difficult location for a forward viewing type endoscope, it is likely that the endoscopy reviewer could not detect any apparent submucosal invasion ( $A$, white arrow). In contrast, the endoscopic ultrasonography (EUS) picture provided a clear image of rough swelling and irregular cystic changes of the third layer (B). Histological examination (C) confirmed its submucosal invasion, which corresponded well to the EUS findings.

\section{Results}

From the results of the blind study, the overall accuracy rates in staging of the invasion depth of early gastric cancer were $63 \%$ for endoscopy and $71 \%$ for EUS. No statistically significant differences were observed. Causes of staging errors significantly differed between endoscopy and EUS. EUS tended to overstage invasion depth (table 2). Endoscopy showed no distinct tendency for understaging or overstaging. All 16 lesions (100\%) staged as mucosal independently but coincidentally by both endoscopy and EUS were found to be limited to the mucosa on histological examination (table 3).

EUS had an overstaging tendency, and the accuracy was $95 \%$ in 21 of the 22 EUSmucosal lesions. In contrast, the 28 endoscopymucosal lesions included six $(21 \%)$ of histologically confirmed submucosal invasion. Such understaging lowered the accuracy rate of

endoscopy-mucosal lesions to $79 \%$ compared with the high accuracy rate of EUS-mucosal lesions.

Of the 28 EUS-submucosal lesions, 15 were accurately staged (54\%), 12 histologically confirmed mucosal lesions (43\%) were overstaged, and one histologically advanced lesion (4\%) was understaged. Of the 24 endoscopysubmucosal lesions, 11 were accurately staged $(46 \%), 11$ were overstaged histologically confirmed mucosal lesions ( $46 \%$ ), and two were understaged histologically confirmed advanced lesions (8\%).

The errors in $20 \mathrm{MHz}$ EUS resulted mainly from various non-cancerous changes in the third layer (submucosa), such as ulcerous changes, submucosal fibrosis, benign cystic changes, and ultrasound attenuated unclear images. The errors of endoscopy resulted mainly from inadequate interpretation of the 
Table 2 Accuracy rates of invasion depth staging of early gastric cancer by endoscopy and $20 \mathrm{MHz}$ endoscopic ultrasonography (EUS)

\begin{tabular}{|c|c|c|c|c|c|}
\hline & \multicolumn{2}{|c|}{ Endoscopy } & \multicolumn{3}{|l|}{ EUS } \\
\hline & Mucosal & Submucosal & Mucosal & Submucosal & Advanced \\
\hline \multicolumn{6}{|l|}{ Histopathology } \\
\hline Mucosal (33) & 22 & 11 & 21 & 12 & 0 \\
\hline Submucosal (17) & 6 & 11 & 1 & 15 & 1 \\
\hline Advanced (2) & 0 & 2 & 0 & 1 & 1 \\
\hline Total accuracy (\%) & 63 & & 71 & & \\
\hline Understaged (\%) & 15 & & 4 & & \\
\hline Overstaged $(\%)$ & 21 & & 25 & & \\
\hline
\end{tabular}

$\mathrm{n}=52$. Causes of staging errors (under or over) differed significantly between endoscopy and EUS $(\mathrm{p}<0.05)$. EUS tended to overstage the invasion depth.

Table 3 Comparison of invasion depth staging of early gastric cancer between endoscopy and endoscopic ultrasonography (EUS)

\begin{tabular}{|c|c|c|c|}
\hline & \multicolumn{3}{|l|}{$E U S$} \\
\hline & Mucosal & Submucosal & Advanced \\
\hline \multicolumn{4}{|l|}{ Endoscopy } \\
\hline Mucosal & $\mathrm{m}, 16$ & $\begin{array}{l}\mathrm{m}, 6 \\
\mathrm{sm}, 6\end{array}$ & \\
\hline \multirow[t]{2}{*}{ Submucosal } & $\mathrm{m}, 5$ & $\mathrm{~m}, 6$ & \\
\hline & $\mathrm{sm}, 1$ & $\begin{array}{l}\text { sm, } 9 \\
\text { ad, } 1\end{array}$ & $\begin{array}{l}\text { sm, } 1 \\
\text { ad, } 1\end{array}$ \\
\hline
\end{tabular}

$\mathrm{n}=52 . \mathrm{m}$, histologically limited within mucosa; $\mathrm{sm}$, with histologically confirmed submucosal invasion; ad, histologically advanced cancer.

depth of the depression, unevenness of the surface, and ulcerous changes.

\section{Discussion}

Our present blind study confirmed the results of our previous pilot study. ${ }^{19}$ The staging accuracy in early gastric cancer invasion depth was almost $70 \%$ in both endoscopy and EUS. Our accuracy rate of differentiating between mucosal cancer and submucosal cancer by both methods was slightly lower than in other reports. ${ }^{17}{ }^{25}$ Indirect observation using photoprints may have affected the accuracy rates. Use of video tapes may be more effective. However, the accuracy rates of both methods were almost the same as in our pilot study. EUS showed a higher overstaging rate. When both methods coincidentally staged a lesion as mucosal cancer, it was correct.

In the actual diagnostic process, the existence of early gastric cancer is discovered mainly by endoscopy, and histologically confirmed by biopsy. When a cancer lesion is staged as mucosal by endoscopy, it could be a candidate for curative endoscopic treatment. The accumulated data show that early gastric cancer without metastasis can be clinically identified in intestinal types of mucosal cancer. ${ }^{26}$ From our present results, 57\% of endoscopy-mucosal lesions were coincidentally EUS-mucosal. They were correctly staged and the indication for endoscopic treatment could be confirmed. There may be some diagnostic discrepancy between Japanese pathologists and Western pathologists in differentiating definite carcinoma from high grade dysplasia. From a practical viewpoint, complete endoscopic resection would be recommended for such mucosal tumours. ${ }^{27} \mathrm{~A}$ definitive diagnosis of mucosal stage by a combination of endoscopy and EUS may be useful to protect against overtreatment by surgical resection for such borderline cases.

On the other hand, it may be important in the choice of treatment for early gastric cancer that $21 \%$ of endoscopy-mucosal cancers had histologically confirmed submucosal invasion. When the lesions were classified as endoscopymucosal but EUS-submucosal, there were the same numbers of histologically confirmed mucosal lesions and submucosal lesions. The choice of treatment should be made bearing in mind the almost $20 \%$ risk of metastasis for cancer with submucosal invasion. ${ }^{28}$ Extended lymphadenectomy for gastric cancer remains controversial in Western countries. ${ }^{29} 30$ However, when a lesion is staged as endoscopymucosal and EUS-submucosal, the patient should be informed of possible lymph node metastasis before the therapy.

The diagnosis of EUS-mucosal was sufficiently accurate, and more precise analysis of findings of submucosal invasion on EUS are expected. The results of our prospective blind study indicate that the combination of endoscopy and EUS may provide useful information for therapeutic choice for early gastric cancer. EUS is expected to compensate for the understaging of lesions of the endoscopy-mucosal stage. When an early gastric cancer lesion fulfils the endoscopic criteria for endoscopic treatment, EUS is required. A double confirmation of the invasion depth is recommended before deciding on a treatment strategy.

This work was supported by a grant from The Japanese Foundation for Research and Promotion of Endoscopy.

1 DiMagno EP, Buxton JL, Regan PT, et al. Ultrasonic endoscope. Lancet 1980;i:629-31.

2 DiMagno EP, Regan PT, Clain JE, et al. Human endoscopic ultrasonography. Gastroenterology 1982;83:824-9.

3 Nakazawa S, Nakamura T, Yoshino J. Endoscopic ultrasonography. In: Oguro Y, Takagi K, eds. Endoscopic approaches to cancer diagnosis and treatment Tokyo: Japan Scientific Societies Press, 1990:41-56.

4 Caletti G, Ferrari A. Endoscopic ultrasonography. Endoscopy 1996;28:156-73.

5 Nick1 NJ, Bhutani MS, Catalano M, et al and the American Endosonography Club. Clinical implications of endoscopic Endosonography Club. Clinical implications of endoscopic
ultrasound: the American Endosonography Club study. Gastrointest Endosc 1996;44:371-7.

6 Tada M, Murakami A, Karita M, et al. Endoscopic resection of early gastric cancer. Endoscopy 1993;25:445-50.

7 Oguro Y. Recent advances in endoscopic treatment. In: Oguro Y, Takagi K, eds. Endoscopic approaches to cancer diagnosis and treatment Tokyo: Japan Scientific Societies Press, 1990:89-99.

8 Lambert R. Endoscopic treatment of esophagogastric tumors. Endoscopy 1996;28:27-37.

9 Tio TL, Schouwink MH, Cikot RJML, et al. Preoperative TNM classification of gastric carcinoma by endosonography in comparison with the pathological TNM system: a prospective study of 72 cases. Hepatogastroenterology

10 Akahoshi K, Misawa T, Fujishima H, et al. Preoperative evaluation of gastric cancer by endoscopic ultrasound. Gut 1991;32:479-82.

11 Lightdale CJ. Endoscopic ultrasonography in the diagnosis, staging and follow-up of esophageal and gastric cancer. Endoscopy 1992;24 (suppl 1):297-303

12 Grimm H, Binmoeller KF, Hamper K, et al. Endosonography for preoperative locoregional staging of esophageal and gastric cancer. Endoscopy 1993;25:224-30.

3 Dittler HJ, Siewert JR. Role of endoscopic ultrasonography in gastric carcinoma. Endoscopy 1993;25:162-6.

14 Takemoto T, Yanai H, Tada M, et al. Application of ultrasonic probes prior to endoscopic resection of early gastric cancer. Endoscopy 1992;24 (suppl 1):329-33.

15 Yanai H, Fujimura H, Suzumi M, et al. Delineation of the gastric muscularis mucosae and assessment of depth of invasion of early gastric cancer using a 20-megahertz endoinvasion of early gastric cancer using a 20-megahertz endo12 .

16 Yanai $H$, Tada $M$, Karita $M$, et al. Diagnostic utility of 20-megahertz linear endoscopic ultrasonography in early gastric cancer. Gastrointest Endosc 1996;44:29-33. 
17 Sano T, Okuyama Y, Kobori O, et al. Early gastric cancer: endoscopic diagnosis of depth of invasion. Dig Dis Sci

18 Colin-Jones DG, Rösch T, Dittler HJ. Staging of gastric cancer by endoscopy. Endoscopy 1993;25:34-8.

19 Yanai H, Matsumoto Y, Harada T, et al. Endoscopic ultrasonography and endoscopy for staging depth of invasion in early gastric cancer: a pilot study. Gastrointest Endosc 1997;46:212-16.

20 Aibe T, Fuji T, Okita K, et al. A fundamental study of normal layer structure of the gastrointestinal wall visualized by endoscopic ultrasonography. Scand F Gastroenterol 1986;21 (suppl 123):6-15.

21 Kimmey MB, Martin RW, Haggitt RC, et al. Histologic correlates of gastrointestinal ultrasound images. Gastroenterology 1989;96:433-41.

22 Yanai H, Yoshida T, Harada T, et al. Endoscopic ultrasonography of superficial esophageal cancers using a thin ultrasound probe system equipped with switchable radial and linear scanning modes. Gastrointest Endosc 1996;44:578-82.

23 Yanai H, Harada T, Matsumoto Y, et al. In-vitro endosonographic appearance of muscularis mucosae of the esophagus. Endoscopy 1997;29:S49.
24 Japanese Research Society for Gastric Cancer. Fapanese classification of gastric carcinoma. 1st English edn. Tokyo: Kanehara \& Co Ltd, 1995.

25 Yasuda K. Endoscopic ultrasonic probes and mucosectomy for early gastric carcinoma. Gastrointest Endosc 1996;43: S29-31.

26 Yamao T, Shirao K, Ono H, et al. Risk factors for lymph node metastasis from intramucosal gastric carcinoma. Cancer 1996;77:602-6.

27 Schlemper RJ, Itabashi M, Kato Y, et al. Differences in diagnostic criteria for gastric carcinoma between Japanese and Western pathologists. Lancet 1997;349:1725-9.

28 Ichikura T, Uefuji K, Tomimatsu S, et al. Surgical strategy for patients with gastric carcinoma with submucosal invasion. Cancer 1995;76:935-40.

29 Lisborg P, Jatzko G, Horn M, et al. Radical surgery (R2 resection) for gastric cancer: a multivariate analysis. Scand f Gastroenterol 1994;29:1024-8.

30 Bonenkamp JJ, Songun I, Hermans J, et al. Randomized comparison of morbidity after D1 and D2 dissection for gastric cancer in 996 Dutch patients. Lancet 1995;345:7458 . 